\title{
Neutral interstellar hydrogen in the inner heliosphere under the influence of wavelength-dependent solar radiation pressure
}

\author{
S. Tarnopolski and M. Bzowski
}

Space Research Centre, Polish Academy of Sciences, Bartycka 18A, 00-716 Warsaw, Poland

e-mail: [slatar;bzowski]@cbk.waw.pl

Received 5 January 2007 / Accepted 10 October 2008

\section{ABSTRACT}

\begin{abstract}
Context. With the plethora of detailed results from heliospheric missions such as Ulysses and SOHO and at the advent of the first mission dedicated to in situ studies of neutral heliospheric atoms IBEX, we have entered the era of precision heliospheric studies. Interpretation of these data requires precision modeling, with second-order effects quantitatively taken into account.

Aims. We study the influence of the non-flat shape of the solar Lyman- $\alpha$ line on the distribution of neutral interstellar hydrogen in the inner heliosphere and assess the importance of this effect for interpretation of heliospheric in situ measurements.

Methods. Based on available data, we (i) constructed a model of evolution for the solar Lyman- $\alpha$ line profile with solar activity; (ii) modified an existing test-particle code used to calculate the distribution of neutral interstellar hydrogen in the inner heliosphere so that it takes the dependence of radiation pressure on radial velocity into account; and (iii) compared results of the old and new version. Results. Discrepancies between the classical and Doppler models appear between $\sim 5$ and $3 \mathrm{AU}$ and increase towards the Sun from a few percent to a factor of 1.5 at $1 \mathrm{AU}$. The classical model overestimates the density everywhere except for a $\sim 60^{\circ}$ cone around the downwind direction, where a density deficit appears. The magnitude of the discrepancies appreciably depends on the phase of the solar cycle, but only weakly on the parameters of the gas at the termination shock. For in situ measurements of neutral atoms performed at $\sim 1 \mathrm{AU}$, like those planned for IBEX, the Doppler correction will need to be taken into account, because the modifications include both the magnitude and direction of the local flux by a few $\mathrm{km} \mathrm{s}^{-1}$ and degrees, respectively, which, when unaccounted for, would introduce an error of a few $\mathrm{km} \mathrm{s}^{-1}$ and degrees in determination of the magnitude and direction of the bulk velocity vector at the termination shock.

Conclusions. The Doppler correction is appreciable for in situ observations of neutral H populations and their derivatives performed a few AU from the Sun.
\end{abstract}

Key words. Sun: UV radiation - ultraviolet: solar system - interplanetary medium - line: profiles - ISM: atoms

\section{Introduction}

Development of models of the distribution of neutral interstellar hydrogen in the heliosphere began after the discovery of a diffuse interplanetary Lyman- $\alpha$ glow (Thomas \& Krassa 1971; Bertaux \& Blamont 1971), interpreted by Fahr (1968) and Blum \& Fahr (1970) as caused by the scattering of solar Lyman- $\alpha$ radiation on this gas flowing through the Solar System.

At the very early phase, the influence of processes going on at the heliospheric interface on the heliospheric gas was neglected. It was assumed that both the ionization due to solar output and the radiation pressure acting on the $\mathrm{H}$ atoms are stationary and spherically symmetric around the Sun and that their rates fall off proportionally to inverse square of heliocentric distance. It was further assumed that the inflowing neutral gas is monoenergetic, i.e., that before the encounter with the Sun the atoms move with identical, parallel-oriented velocities, equal to the macroscopic bulk velocity of the gas.

These assumptions formed the basis of the first model of density distribution of neutral interstellar gas around the Sun: the purely analytical "cold" model (Fahr 1968; Axford 1972). It features axial symmetry about the axis of inflow of the gas and shows either a singularity at the downwind axis, when radiation pressure is too weak to compensate for solar gravity, or an empty "avoidance zone" in the downwind region with a paraboloidal boundary surface, when radiation pressure overcompensates for solar gravity.

Lifting of the monoenergetic assumption (i.e. allowing for a finite temperature of interstellar gas, high enough to yield thermal velocity comparable to the bulk velocity of the gas), adoption of the distribution function of the gas far away from the Sun ("in infinity") in the form of a Maxwellian shifted in the velocity space by the bulk velocity vector, and assumption that the gas is collisionless on the distance scale comparable to the size of the heliosphere, all allowed us to use the Boltzmann equation to describe the problem of the interaction of neutral interstellar gas with the solar environment. Its solution brought the "hot model" of the gas distribution in the inner heliosphere (Thomas 1978; Fahr 1978, 1979; Wu \& Judge 1979; Lallement et al. 1985a). This model required numerical integration of the distribution function, but the function itself was still analytical. The "hot model" in its various implementations became the canonical model of neutral interstellar gas distribution in the inner heliosphere, so further on we will refer to it as "the classical hot model". 
Further modeling of the interaction and distribution of neutral interstellar hydrogen near the Sun focused on two main topics. On one hand, a lot of effort was made toward simulating and understanding processes going on at the boundary between the expanding solar wind and the incoming, partially ionized interstellar gas, in the region referred to as the heliospheric interface - and this issue will not be addressed in the present paper: the reader is referred to recent reviews by Baranov (2006b,a); Izmodenov (2006); Izmodenov \& Baranov (2006). On the other hand, development of the hot model continued, aimed at a more realistic description of the distribution of neutral interstellar hydrogen in the inner heliosphere, suitable for quantitative, and not only qualitative interpretation of heliospheric measurements.

In the first shot, the assumption that the ionization rate is spherically symmteric was eliminated, when Lallement et al. (1985b) described the latitudinal modulation of the charge exchange rate with a one-parameter formula $1-A \sin ^{2} \phi$, implementing it in the CNRS model of the heliospheric gas distribution. This allowed the authors to vary the equator-to-pole contrast of the ionization rate, but required keeping the width and range of the enhanced ionization band fixed.

A different extension of the hot model was proposed by Ruciński \& Fahr (1989, 1991), who realized that the rate of ionization by electron impact is not proportional to the inverse square of solar distance. Electron ionization is particularly important for interstellar helium (McMullin et al. 2004; Lallement et al. 2004; Witte 2004). The effects in hydrogen are only noticeable within a few AU from the Sun, where the density of hydrogen gas is already very reduced by earlier ionization and solar radiation pressure, overcompensating for solar gravity; therefore this aspect of the physics of neutral interstellar hydrogen has been neglected for quite a while since then, but is reintroduced in the most recent versions of the model (Bzowski et al. 2008).

In the next round in the development of the density model, gone was assumption of the invariability of radiation pressure and ionization rate. This aspect of the physics of neutral interstellar gas was implemented in a simplified numerical model by Kyrölä et al. (1994) and shortly afterwards in the Warsaw test-particle code, to which we will refer in this paper as the Warsaw model (Ruciński \& Bzowski 1995a; Bzowski \& Ruciński 1995a,b; Bzowski et al. 1997). Indeed, both the solar EUV flux (Floyd et al. 2002) and the solar wind flux (King \& Papitashvili 2005) vary considerably during the solar cycle. The result is a solar cycle variation of the solar radiation pressure, of the EUV ionization rate, and of the rate of charge exchange between neutral $\mathrm{H}$ atoms and solar wind protons. This in turn results in appreciable variations in the density and bulk velocity of neutral interstellar hydrogen within a dozen AU from the Sun.

The Warsaw time-dependent model was fully numerical: not only did the integration of the distribution function have to be performed numerically, as in the classical "hot model", but also the calculation of the integrand, i.e. of the distribution function itself, was no longer analytical. At this phase in the heliospheric research, substitute idealized models of the evolution of these parameters had to be used due to the lack of sufficiently long time series of measurements of the solar wind speed and density, and of the solar EUV output. These aspects of the development of heliospheric gas models were discussed in greater detail in a review by Ruciński \& Bzowski (1996).

In the next move, the inner-heliospheric effects of the interaction of the solar wind and interstellar gas within the heliospheric interface were taken into account. Owing to the charge exchange between the atoms of interstellar gas and the heated and compressed plasma in front of the heliopause, the original population of neutral atoms is somewhat cooled and accelerated, and a new population of atoms appears. These new neutral atoms initially maintain the properties of the parent plasma population, but later on they decouple from this plasma and flow through the heliopause and inside the termination shock of the solar wind. The two neutral populations interact further with the ionized components, exchanging charge with the protons from the local plasma and producing new populations of neutral atoms. Hence, the processes in the heliospheric interface create a few distinct, collisionless populations of neutral atoms (Osterbart \& Fahr 1992; Baranov et al. 1991; Baranov \& Malama 1993), as discussed in detail by Izmodenov (2000) and Malama et al. (2006).

From the viewpoint of modeling the distribution of neutral interstellar hydrogen in the inner heliosphere, the most important aspect of the processes going on in the heliospheric interface is the modification of the distribution function at the termination shock (Izmodenov et al. 2001). Instead of the shifted Maxwellian with parameters homogeneous in space, Scherer et al. (1999) adopted a sum of two Maxwellians, with nonisotropic temperatures, shifted by appropriate bulk velocity vectors. The two components of the new functions corresponded to the two thermal populations (primary and secondary) predicted by the Moscow Monte Carlo simulation of heliospheric interface and had parameters that depended on the offset angle $\theta$ from the upwind direction. This new version of the Warsaw model was time dependent, but still axially symmetric. At that time, enough measurements had been published to attempt to introduce observation-based models of the radiation pressure and ionization rate to the simulations.

Axial symmetry was removed in the next round of the Warsaw model development, when an anisotropy of the ionization rate was introduced. Bzowski et al. (2001, 2002) allowed the ionization rate to change as a continuous function of the heliographic latitude, with the latitudinal profile of the ionization rate continuously changing with the phase of solar cycle. As a result, the ionization field in the model became 2D (maintainining the axial symmetry about the solar rotation axis) and time-dependent; and since the gas inflow axis and solar rotation axis are inclined at an angle to each other, the model of neutral hydrogen distribution in the inner heliosphere became 3D and time-dependent.

The most recent extension of the Warsaw model is presented in this paper. We improve on the modeling of radiation pressure acting on individual $\mathrm{H}$ atoms, which now is not only a function of time, but also of the radial velocity of the atom. In the previous versions of the model it was assumed that the profile of the solar Lyman- $\alpha$ line, responsible for the radiation pressure, is flat. In reality it not only is not flat, but shows considerable variations depending on the phase of solar activity (Lemaire et al. 2002; Lemaire et al. 2005).

We take this into account, and in Sect. 2 we develop an observation-based model of the evolution of the line profile as a function of the wavelength- and disk-integrated flux. We use this model in a newly-developed code that simulates the density and higher moments of the distribution function of neutral interstellar hydrogen in the inner heliosphere. We discuss these calculations in Sect. 3. With the use of the newly-developed code, we assess modifications of the local gas density and flux with respect to the results of models neglecting the dependence of radiation pressure on the radial velocity. We also show possible implications for interpretation of heliospheric in situ measurements of neutral atoms and pickup ions. This discussion is provided in Sect. 4. Section 5 offers a summary of the results. 


\section{The Doppler model of neutral hydrogen distribution in the inner heliosphere}

The approach exercised in the present model is a modification of the approach presented by Ruciński \& Bzowski (1995a). The density and higher moments of the distribution function of neutral interstellar hydrogen at a location $\boldsymbol{R}$ in the inner heliosphere at a time $\tau$ are calculated by numerical integration of the local distribution function, constructed as a product of the distribution function in the source region $w_{\text {src }}$ and of the probability $w_{\text {ion }}$ of survival of the atoms traveling from the source region to the local point $\boldsymbol{R}$ against ionization,

$f(\boldsymbol{R}, \boldsymbol{V}, \tau)=w_{\mathrm{src}}\left(\boldsymbol{r}_{\mathrm{src}}(\boldsymbol{R}, \boldsymbol{V}, \tau), \boldsymbol{v}_{\mathrm{src}}(\boldsymbol{R}, \boldsymbol{V}, \tau)\right) w_{\mathrm{ion}}(\boldsymbol{R}, \boldsymbol{V}, \tau)$

where $\boldsymbol{r}_{\mathrm{src}}(\boldsymbol{R}, \boldsymbol{V}, \tau)$ is the start position in the source region of the atom that at the local point $\boldsymbol{R}$ at time $\tau$ has velocity $\boldsymbol{V}$, and $\boldsymbol{v}_{\text {src }}(\boldsymbol{R}, \boldsymbol{V}, \tau)$ is the relevant start velocity in the source region.

From the numerical point of view, the distribution function in the source region $w_{\text {src }}$ can a priori be any reasonable function. In the following we adopted it either as a Maxwellian shifted by the bulk velocity vector, homogeneous in space and independent of time,

$w_{\mathrm{src}}\left(\boldsymbol{r}_{\mathrm{src}}, \boldsymbol{v}_{\mathrm{src}}\right)=n_{\mathrm{src}}\left(\frac{m}{2 \pi k T_{\mathrm{src}}}\right)^{3 / 2} \exp \left(-\frac{m\left(\boldsymbol{v}_{\mathrm{B}}-\boldsymbol{v}_{\mathrm{src}}\right)^{2}}{2 k T_{\mathrm{src}}}\right)$

or, in the two-populations case, as a sum of two such functions with different parameters $n_{\text {src }}$ (density in the source region), $\boldsymbol{v}_{\mathrm{B}}$ (bulk velocity at the source region), and $T_{\text {src }}$ (temperature at the source region); also, $m$ is the atom mass, and $k$ the Boltzmann constant. Basically, the parameters of these populations are functions of the location in the heliospheric interface (Izmodenov et al. 2001). Since our goal in this paper is to investigate the effect of the $v_{\mathrm{r}}$-dependence of radiation pressure on the gas distribution, we left this problem out of the analysis. Some insight into modifications of the gas density profile due to the angular gradient of the parameters of the $\mathrm{H}$ populations at the termination shock is provided by Bzowski et al. (2008).

The challenging issue in calculating the local distribution function of the gas is finding the link between the local velocity of the test atom $\boldsymbol{V}$ at time $\tau$ and location $\boldsymbol{R}$ and its location $\boldsymbol{r}_{\mathrm{src}}$ and velocity $\boldsymbol{v}_{\text {src }}$ in the source region in a scenario where the radiation pressure is a function of time and radial velocity. Indeed, the force acting on an $\mathrm{H}$ atom in the heliosphere is composed of the attracting solar gravity and the repelling radiation pressure, which is a function of the spectral flux $I_{\lambda}$ relevant for a given radial velocity $v_{\mathrm{r}}$. The spectral flux $I_{\lambda}$ is also a function of the line-integrated flux $I_{\text {tot }}(t)$, which varies on time scales from days to dozens of years. Thus the spectral flux responsible for the instantaneous radiation pressure acting on an $\mathrm{H}$ atom, moving with radial velocity $v_{\mathrm{r}}$, is a function of $v_{\mathrm{r}}, I_{\mathrm{tot}}(t)$ and, implicitly, of $t$ : $I_{\lambda}=I_{\lambda}\left(v_{\mathrm{r}}, I_{\text {tot }}(t)\right)$.

Since both $I_{\text {tot }}$ and solar gravity fall off with the heliocentric distance as $1 / r^{2}$, the radiation pressure can be expressed by a factor $\mu$ of compensation of the solar gravity. In the modeling, this factor is a function of the radial velocity and line-integrated flux, which in turn is a function of time; hence, finally $\mu=\mu\left(v_{\mathrm{r}}, t\right)$.

The link between the local position and velocity vectors $\boldsymbol{R}, \boldsymbol{V}$ and the corresponding position and velocity vectors at the source region $\boldsymbol{r}_{\text {src }}, \boldsymbol{v}_{\text {src }}$ is determined by solving the equation of motion specified in Eq. (3), performed numerically backwards in time.
Along with the calculation of the trajectory of the atom, its probability of survival is calculated.

$$
\frac{\mathrm{d}}{\mathrm{d} t}\left(\begin{array}{c}
x \\
y \\
z \\
v_{x} \\
v_{y} \\
v_{z} \\
u \\
u_{\beta}
\end{array}\right)=\left(\begin{array}{c}
v_{x} \\
v_{y} \\
v_{z} \\
-\frac{G M\left(1-\mu\left(t, v_{\mathrm{r}}\right)\right)}{G M\left(1-\mu\left(t, v_{\mathrm{r}}\right)\right)} \\
-\frac{G\left(r^{3}\right.}{\left.r^{3}\left(t, v_{\mathrm{r}}\right)\right)} z \\
-\frac{G M\left(r^{3}\right.}{\frac{1}{r}^{2}} \\
u \beta_{0}(r, t, \phi)
\end{array}\right) .
$$

In the equation above, $\beta_{0}(r, t, \phi)=r^{2} \beta(r, t, \phi)$ is the scaled ionization rate at a heliographic latitude $\phi, r^{2}=x^{2}+y^{2}+z^{2}$, $G M$ is the constant of gravity times the solar mass, and $v_{r}(t)=$ $(\boldsymbol{r}(t) \cdot \boldsymbol{v}(t)) /|\boldsymbol{r}(t)|$ is the radial velocity of the atom. The position vector $\boldsymbol{r}(t)$ has coordinates $x, y, z$ and the velocity vector $\boldsymbol{v}(t)$ coordinates $v_{x}, v_{y}, v_{z} ; u_{\beta}$ is used to calculate the survival probability as:

$w_{\text {ion }}=\exp \left(-u_{\beta}\right)$.

Once the position and velocity vectors of the test atom in the source region are found (respectively, $\boldsymbol{r}_{\mathrm{src}}, \boldsymbol{v}_{\mathrm{src}}$ ), the value of the distribution function in the source region $w_{\text {src }}$ can be calculated. The value of the local distribution function is then obtained as a product of $w_{\text {src }}$ and the survival probability $w_{\text {ion. }}$. To obtain the density and higher moments, the local distribution function is numerally integrated in the velocity space $\mathrm{d}^{3} V$.

The model of radiation pressure (Tarnopolski 2007) was developed based on a series of observations of the solar Lyman- $\alpha$ line profiles, performed by SUMER/SOHO between solar minimum and maximum (Lemaire et al. 2002) and available on the Web. Since the SUMER instrument is located at the SOHO spacecraft orbiting around the L1 Lagrange point between the Earth and the Sun (Wilhelm et al. 1999), the observations are free of the geocoronal contamination that affected earlier measurements carried out from low-Earth orbits (e.g. OSO-5, Vidal-Madjar 1975). The absolute calibration of the wavelength reported by Lemaire et al. (2002) is better than $\pm 0.0015 \mathrm{~nm}$. The calibration of intensity was performed by direct comparison of results of integrating the observed profiles with the absolute fluxes obtained with the SOLSTICE instrument. The accuracy of the net flux was reported at a $\pm 10 \%$ level. It seems, however, that the data published on the Web are a smoothed version of actual measurements because the data points within the profiles do not show any scatter in spite of the accuracy of $10 \%$, reported in the paper.

Fitting the functional forms of the profiles used in earlier studies (Fahr 1978; Chabrillat \& Kockarts 1997; Scherer et al. 2000) did not yield sufficient accuracy of the model (cf. Figs. 1 and 2). To develop the model of $\mu\left(v_{\mathrm{r}}, I_{\mathrm{tot}}\right)$ discussed in this paper, the original data were rescaled to the spectral flux in the $\mu$ units as a function of radial velocity in $\mathrm{km} \mathrm{s}^{-1}$. A satisfactory result could be obtained with a 9-parameter function in the form (Tarnopolski 2007)

$$
\begin{aligned}
\mu\left(v_{\mathrm{r}}, I_{\mathrm{tot}}(t)\right)= & A\left[1+B I_{\mathrm{tot}}(t)\right] \exp \left(-C v_{\mathrm{r}}^{2}\right) \\
& \times\left[1+D \exp \left(F v_{\mathrm{r}}-G v_{\mathrm{r}}^{2}\right)+H \exp \left(-P v_{\mathrm{r}}-Q v_{\mathrm{r}}^{2}\right)\right]
\end{aligned}
$$

with the following parameters:

$$
\begin{aligned}
& A=2.4543 \times 10^{-9}, B=4.5694 \times 10^{-4}, C=3.8312 \times 10^{-5} \\
& D=0.73879, \quad F=4.0396 \times 10^{-2}, \quad G=3.5135 \times 10^{-4} \\
& H=0.47817, \quad P=4.6841 \times 10^{-2}, \quad Q=3.3373 \times 10^{-4}
\end{aligned}
$$




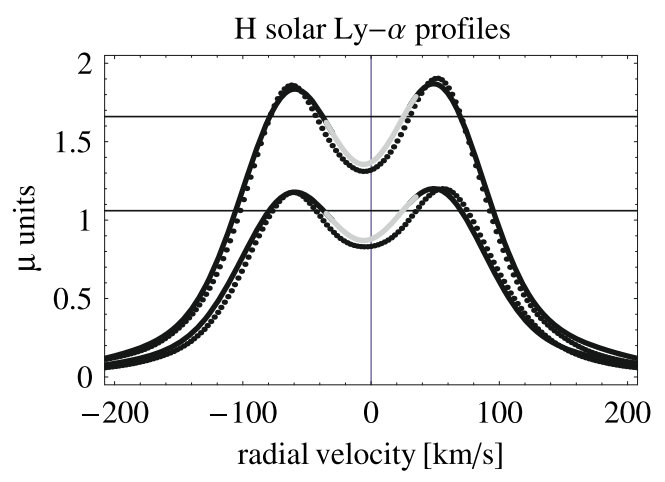

Fig. 1. Selected profiles of the solar Lyman- $\alpha$ line observed by SUMER/SOHO (dots) for solar minimum (lower line, Feb. 17, 1997) and solar maximum (upper line, May 20, 2000), compared with the results of the fitted model, specified in Eq. (5) (lines). The gray part of the model lines corresponds to $\pm 30 \mathrm{~km} \mathrm{~s}^{-1}$ around 0 and illustrates the range on the profile that is the most relevant for the thermal populations of neutral $\mathrm{H}$ atoms in the heliosphere. The horizontal lines mark the $\mu$ values used in the comparison simulations with the use of the classical hot model.

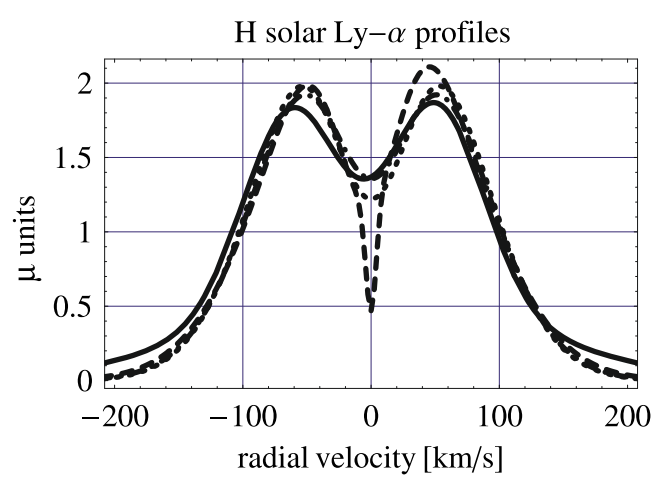

Fig. 2. Comparison of models of the solar Lyman- $\alpha$ line profile: solid line: the present model; dashed line: Chabrillat \& Kockart; dashed-dot line: Fahr et al.; dots: Scherer.

An interesting aspect of the model is that its sole solar-related parameter is the line-integrated flux in the Lyman- $\alpha$ line $I_{\text {tot }}$. Any dependence of the radiation pressure on time goes to the model via $I_{\text {tot }}$. Hence, with a model of the behavior of $I_{\text {tot }}$ in time in hand (such as, e.g., discussed by Bzowski 2001; and Bzowski et al. 2008) we can immediately construct a model of the radiation pressure that will depend on the radial velocity of the atoms as well.

The function defined in Eq. (5) with the parameters as listed above reproduces the observed profiles well both for solar minimum (the profiles from July 27, 1996 to August 24, 1997) and solar maximum (the profiles from August 20, 1999 to August 22, 2001). The accuracy of the fit for the most interesting region $\pm 140 \mathrm{~km} \mathrm{~s}^{-1}$ about the line center exceeds the accuracy of observations declared by the authors. Furthermore, the lineintegrated fluxes obtained from the model profiles agree with the line-integrated data and with the total fluxes reported by Tobiska et al. (2000) for a given day. The differences are around 5\%. An exception is the profile observed on May 20, 2000, for which the difference is $9 \%$, still within the absolute calibration accuracy of $10 \%$. A comparison of the experimental data with the fitted model is shown in Fig. 1. A comparison of this model with earlier models (Fahr et al. 1981; Chabrillat \& Kockarts 1997; Scherer et al. 2000) is presented in Fig. 2.
Since the absolute calibration of $I_{\text {tot }}$ has changed since the time of publication of the profiles, we show the original profiles recalibrated by multiplying by an appropriate factor so that their absolute fluxes agree with the values accepted nowadays. The differences between the models in the velocity range $\pm 100 \mathrm{~km} \mathrm{~s}^{-1}$ around the line center are around 10 to $15 \%$. For higher velocities, the percentage differences between the models are not as important because the absolute values of radiation pressure are low anyway; furthermore, the fast atoms are not very sensitive to the radiation pressure in general (Bzowski 2008). The highest differences in the absolute terms occur at the peaks of the profile, i.e., at the velocities characteristic of the fast wing of the distribution function of thermal populations of neutral hydrogen in the inner heliosphere. With as many as 9 data sets available, covering the entire span of solar activity levels, we were able to come up with a model that seems to reproduce physical reality better than the former ones.

Our model does not feature any nonlinear response of the line profile to $I_{\text {tot }}$, although such a character of the response might be expected based on the findings of Amblard et al. (2008) and Emerich et al. (2005). Amblard et al. show that the spectrum of the Sun in the EUV region is composed of three components whose contributions to the net sprectral flux vary with the phase of the solar cycle, and they try to attribute these components to different features at the solar surface and in the solar atmosphere. Since the distribution of these features is a function of heliolatitude, one might then expect that the shape of the solar Lyman- $\alpha$ profile should vary with heliolatitude, but when lacking actual data, a construction of such a model at present is impossible. Emerich et al. presented a nonlinear fit of the central value of the solar spectral flux in the Lyman- $\alpha$ line as a function of the net flux $I_{\text {tot }}$, but within the $I_{\text {tot }}$ range of $3.3 \times 10^{11}$ to $6.5 \times 10^{11} \mathrm{~cm}^{-2} \mathrm{~s}^{-1}$, deviations in their formula from a purelylinear dependence do not exceed $2 \%$, which is much less than all the uncertainties of the profile and $I_{\text {tot }}$ measurements. Hence we believe the model we use in this paper is a reasonable approximation, given the accuracy of available observations.

The numerical scheme presented in this section was devised to enable calculating the density and higher moments of the distribution function in the case of a fully $3 \mathrm{D}$ and time-dependent radiation pressure and ionization rate. The code has already been used in its full mode by Tarnopolski \& Bzowski (2008) to assess the flux of interstellar deuterium at the Earth orbit and by Bzowski et al. (2008) to investigate the density of neutral interstellar $\mathrm{H}$ at the termination shock based on the observations of hydrogen pickup ions by Ulysses. In the present study, the code was restricted to the spherically symmetric and stationary case to facilitate an assessment of the influence of the $v_{\mathrm{r}}$-dependence of the radiation pressure on the distribution of interstellar hydrogen in the inner heliosphere, unblended with other departures from the classical hot model.

\section{Calculations}

The simulations were performed in two groups. In the first one, we investigated the differences between the classical hot model and the Doppler model in a scenario with two populations at the termination shock: the primary interstellar population and the secondary population, which comes up between the heliopause and the bow shock from charge exchange between the atoms from the original interstellar populations and the local heated and compressed plasma. These results are presented in Sect. 4.1 and will be referred to as the composite model, in the sense that it is composed of the two populations: primary and secondary. 

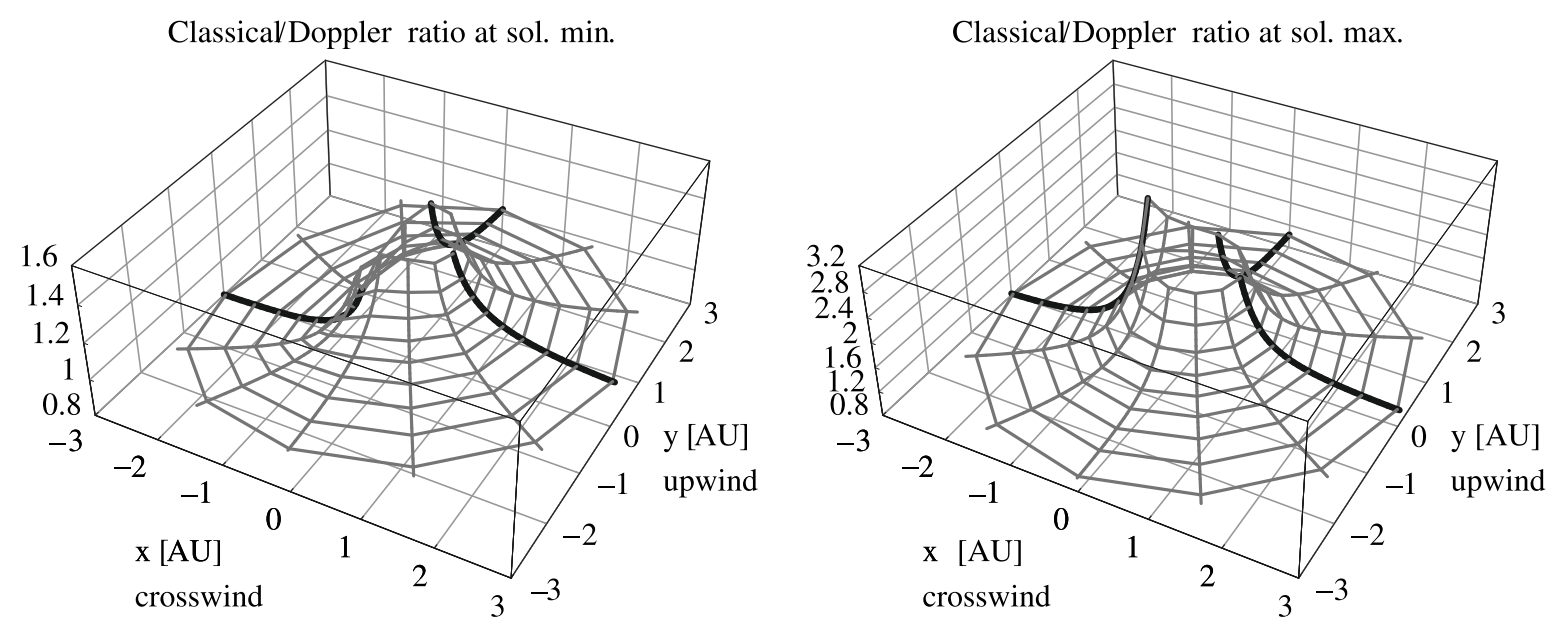

Fig. 3. Classical/Doppler density ratios in the composite model for the solar minimum (left panel) and solar maximum conditions (right panel). The Sun is at the center in the $(0,0)$ point, the upwind - downwind direction is along the $y=0$ line. The upwind, crosswind, and downwind axes are marked with heavy lines.

In the second group of simulations, we checked the robustness of the conclusions drawn in Sect. 4.1 against uncertainties in the bulk velocity and temperature of interstellar gas at the termination shock and against uncertainties in the ionization rate in the inner heliosphere. The results of this series are discussed in Sect. 4.2.

The calculations were performed on a mesh of heliocentric distances $r$ equal to $0.4,0.7,1,2,4,7,10,20,50$, and $100 \mathrm{AU}$ and of offset angles from the upwind directions $\theta$ equal to $0^{\circ}$, $30^{\circ}, 60^{\circ}, 90^{\circ}, 120^{\circ}, 150^{\circ}$, and $180^{\circ}$, separately for solar minimum and solar maximum conditions, characterized by the following values of the line-integrated solar Lyman- $\alpha$ flux and net ionization rate:

solar max: $I_{\mathrm{tot}}=5.50 \times 10^{11}$ photons $\mathrm{cm}^{-2} \mathrm{~s}^{-1}, \beta=8 \times 10^{-7} \mathrm{~s}^{-1}$ solar min: $I_{\text {tot }}=3.53 \times 10^{11}$ photons $\mathrm{cm}^{-2} \mathrm{~s}^{-1}, \beta=5 \times 10^{-7} \mathrm{~s}^{-1}$. The boundary conditions of the composite simulation were the parameters returned by the Moscow MC model at the nose of the termination shock for the following set of LIC conditions (Izmodenov et al. 2003): proton density $n_{\mathrm{p}, \mathrm{LIC}}=0.06 \mathrm{~cm}^{-3}$, neutral $\mathrm{H}$ density $n_{\mathrm{H}, \mathrm{LIC}}=0.18 \mathrm{~cm}^{-3}$, temperature of the gas $T_{\text {LIC }}=6400 \mathrm{~K}$, bulk velocity $v_{\mathrm{B}, \mathrm{LIC}}=26.4 \mathrm{~km} \mathrm{~s}^{-1}$, density of $\mathrm{He}^{+}$in the LIC $n_{\mathrm{He}^{+}}=0.008 \mathrm{~cm}^{-3}$.

The resulting parameters of the primary and secondary populations at the nose of the termination shock were the following: Primary: $n_{\mathrm{TS} \text {,pri }}=0.03465 \mathrm{~cm}^{-3}, v_{\mathrm{TS} \text {,pri }}=28.512 \mathrm{~km} \mathrm{~s}^{-1}$, $T_{\mathrm{TS}, \text { pri }}=6020 \mathrm{~K}$;

Secondary: $n_{\mathrm{TS}, \mathrm{sec}}=0.06021 \mathrm{~cm}^{-3}, v_{\mathrm{TS}, \mathrm{sec}}=18.744 \mathrm{~km} \mathrm{~s}^{-1}$, $T_{\mathrm{TS}, \mathrm{sec}}=16300 \mathrm{~K}$.

These parameters were adopted as the boundary conditions for the composite simulations, performed with the use of the Warsaw model.

In addition to the dependence of radiation pressure on $v_{\mathrm{r}}$, the code also included a simplified model of the electron ionization rate, as adopted by Bzowski et al. (2008). The model is spherically symmetric and identical for the solar minimum and maximum conditions. Inclusion of this model is a departure from the approach adopted in this study to keep all aspects of the modeling as close to the classical hot model as possible. We decided to include the electron ionization to make certain that we do not miss possible disturbances of the Doppler-to-hot model ratios due to the extra ionization term that does not conform with the $1 / r^{2}$ distance dependence of the charge exchange and photoionization rates.
The simulations were repeated with the use of a model that differs from the former one only by the lack of sensitivity of the radiation pressure to $v_{\mathrm{r}}$, i.e. effectively using the classical hot model with the electron ionization term added. The radiation pressure was equal to the (appropriately scaled) spectral flux averaged by $\pm 30 \mathrm{~km} \mathrm{~s}^{-1}$ about 0 , as indicated in Fig. 1 . The numerical accuracy of the solutions was at the level of 1 to $2 \%$.

The simulations discussed in Sect. 4.2 assume there is only one population of interstellar hydrogen at the termination shock. We explored the parameter space adopting the net ionization rates at $1 \mathrm{AU} \beta$, bulk velocities $v_{\text {src }}$, and temperatures $T_{\text {src }}$ covering the entire range of expected values from the low values of $\beta$ relevant for the fast solar wind conditions and solar minimum to the high values relevant for slow solar wind conditions and solar maximum, the ranges of $v_{\text {src }}$ and $T_{\text {src }}$ from the extremes predicted for the primary and secondary populations at the termination shock.

This scan over $\beta$ was performed by assuming that the gas temperature and velocity at the termination shock are equal, respectively, to $12500 \mathrm{~K}$ and $22 \mathrm{~km} \mathrm{~s}^{-1}$, as derived by Costa et al. (1999) from an analysis of the heliospheric Lyman- $\alpha$ glow in the upwind hemisphere, for $I_{\text {tot }}$ relevant for solar minimum and maximum conditions(see above). The scan over $v_{\text {src }}$ and $T_{\text {src }}$ space assumed that $\beta$ and $I_{\text {tot }}$ values are as listed for solar minimum and maximum conditions.

The baseline set of simulations used the newly-developed Doppler model. Comparison simulations used the classical hot model on identical spatial mesh and assumed identical parameters at the termination shock and an identical ionization rate. In this section the electron ionization was not included, so the ionization rate was fell off as $1 / r^{2}$.

\section{Results}

\subsection{Composite model}

The simulations show that neglecting of the $v_{\mathrm{r}}$-dependence of radiation pressure produces an excess of local hydrogen density (see Fig. 3). The excess $q$ is defined here as the ratio of density returned by the classical hot model $n_{\text {class }}$ to the density returned by the Doppler model $n_{\text {dopp }}: q=n_{\text {class }} / n_{\text {dopp }}$. Discrepancies between the classical and Doppler models appear at $\sim 3 \mathrm{AU}$ and increase towards the Sun. Depending on the phase of the solar 

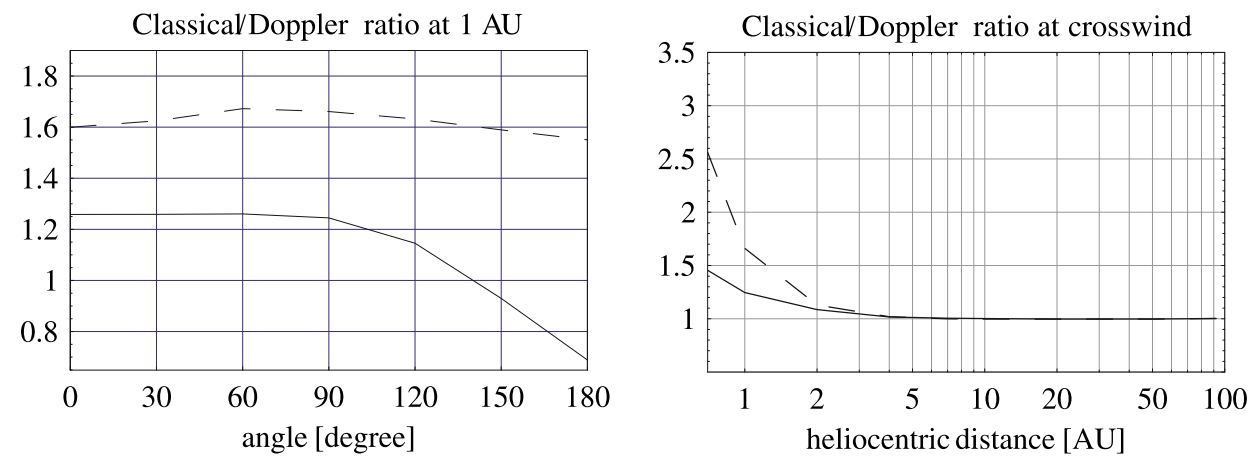

Fig. 4. Density excess $q$ of neutral interstellar hydrogen in the inner heliosphere, defined as a ratio of results of the classical hot model to the results of the Doppler model. Left panel: the density ratio at $1 \mathrm{AU}$ as a function of the offset angle from the upwind direction, right panel: the density ratio at crosswind as a function of the heliocentric distance. Solid lines correspond to the solar minimum conditions, and broken lines to the solar maximum conditions.

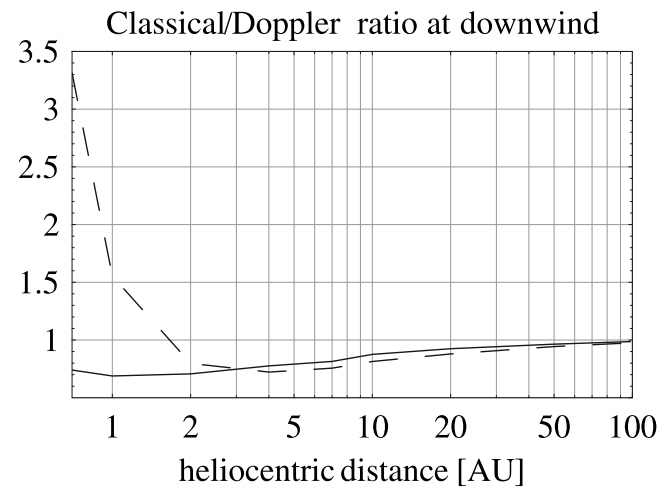

Fig. 5. Density ratio $q$ of the classical hot model to the Doppler model at the downwind axis as a function of the heliocentric distance. Solid line: solar minimum conditions, broken line - solar maximum conditions.

cycle and the offset angle from upwind, at 1 AU they range from a few dozen percent up to a factor of $\sim 1.7$ (see Fig. 4). The excess density is a strong function of solar activity; while at solar minimum its typical values at $1 \mathrm{AU}$ are about $25 \%$, they are a little more than twice as high at solar maximum. An exception is a conical region around the downwind axis with the opening angle of $\sim 60^{\circ}$, as shown in Figs. 4 and 5. On the downwind axis, the hot model predicts a density deficit, which almost does not depend on the activity phase except at the closest distances to the Sun. During solar maximum the excess is predicted even in the downwind region, though only for distances under $\sim 1.5 \mathrm{AU}$.

The existence of the density excess can be understood easily after inspecting the shape of the solar Lyman- $\alpha$ line profile shown in Fig. 1. Since the profile has a minimum close to 0 radial velocity, the $\mathrm{H}$ atoms before approaching the Sun sense a stronger repulsion than in the case when one adopts a "flat" radiation pressure with the mean value averaged between $\pm 30 \mathrm{~km} \mathrm{~s}^{-1}$ around the line center. As a result, in the Doppler case a larger portion of these atoms will be slowed down and repelled from the Sun, which reduces the density in comparison with the classical case. The ensemble of surviving $\mathrm{H}$ atoms has a lower bulk velocity than predicted by the classical model, which leads to a further enhancement of the excess, because the ionization has more time to eliminate the atoms traveling more slowly. On the other hand, a sub-population of atoms exists that on approach towards the Sun experience a lower radiation pressure in the Doppler model than in the classical model. These are the atoms from the slow wing of the distribution function. Although the Doppler model predicts for them less deceleration than the classical model, these atoms contribute comparatively little to the entire ensemble because they are more readily eliminated by ionization than the faster atoms.

The Doppler model predicts different ratios of densities between pairs of offset angles $\theta_{1}, \theta_{2}$ (e.g. upwind/crosswind, upwind/downwind etc.) than the predictions of the classical hot model. The discrepancies are not strong in the upper hemisphere, but escalate with the increase in the difference $\theta_{2}-\theta_{1}$. This must be one of the reasons for which interpreting downwind observations in the heliospheric Lyman- $\alpha$ glow has always been more challenging than in the upwind ones and usually returned different conclusions (e.g. Lallement et al. 1985a; Quémerais et al. 1992). The challenge can be better appreciated when one realizes that the wavelength-sensitivity effects are convolved with the effects related to variations in the solar Lyman- $\alpha$ flux and of the ionization rate, as discussed, e.g., by Ruciński \& Bzowski (1995b); Bzowski \& Ruciński (1995a,b); Bzowski et al. (2002), and with the effects of inhomogeneous distribution of the parameters of interstellar gas along the termination shock (Izmodenov et al. 2001).

The Doppler and classical models predict surprisingly small differences between the local bulk velocities of the gas (Fig. 6). During solar minimum they are about $1 \mathrm{~km} \mathrm{~s}^{-1}$, and they increase to $\sim 2 \mathrm{~km} \mathrm{~s}^{-1}$ during solar maximum. Differences in the radial component of the bulk velocities at $1 \mathrm{AU}$ are also small (Fig. 7). They begin only at the offset angle $\theta \simeq 60^{\circ}$ and increase towards downwind, and the classical model systematically predicts somewhat higher values. Since the differences in $v_{\mathrm{r}}$ are small, we do not expect big differences in the model spectral profiles of the heliospheric glow.

Even though the differences in the local velocities are small, the effect of the wavelength dependence of radiation pressure on the magnitude of fluxes of the two heliospheric neutral H populations is not negligible because of the appreciable differences in the local densities of the gas. As shown in Fig. 9, the classical excess of the flux is 15 to $20 \%$ during solar minimum, depending on the population, and during solar maximum it may reach a factor of 1.4 to 1.9 in the upwind hemisphere and even more in the downwind region, but with the absolute magnitude of the flux reduced by 2 orders of magnitude from the solar minimum level (Fig. 8). In the upwind hemisphere it is very weakly sensitive to the offset angle. The wavelength dependence of radiation pressure must then be appropriately taken into account when interpretating direct in situ observations of neutral interstellar hydrogen atoms, such as those planned for IBEX. The local flux of the atoms is a product of the local velocity vector and of the local 

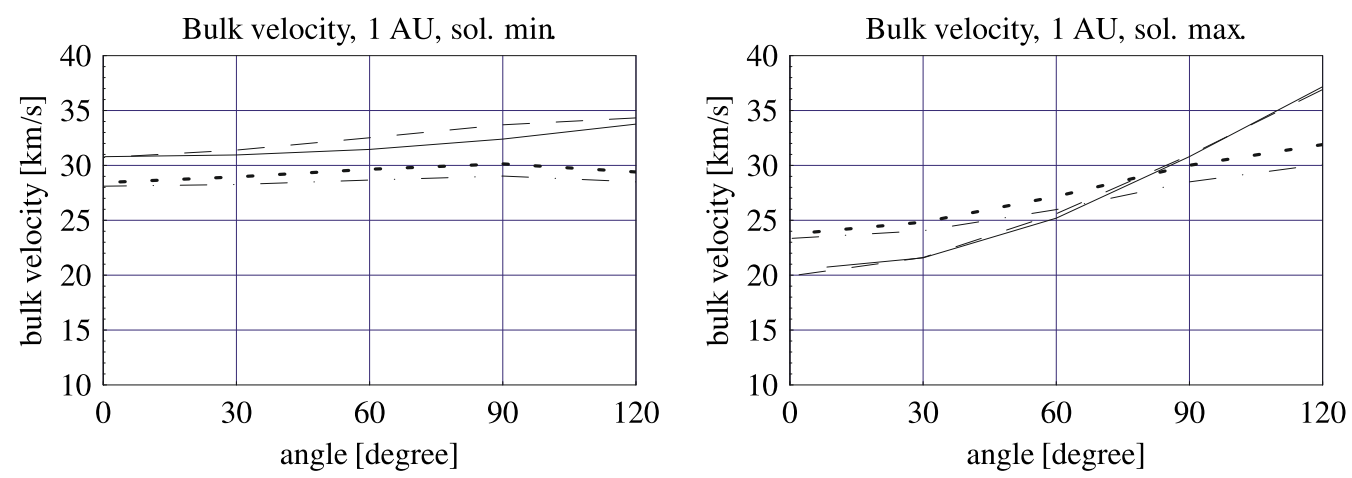

Fig. 6. Bulk velocities of the primary and secondary populations of neutral interstellar $\mathrm{H}$ at $1 \mathrm{AU}$, calculated using the Doppler and classical models, shown as a function of the offset angle from upwind for the solar minimum (left panel) and solar maximum conditions (right panel). Primary population: solid line (classical) and broken line (Doppler); secondary population: dotted line (Doppler) and dash-dot (classical).
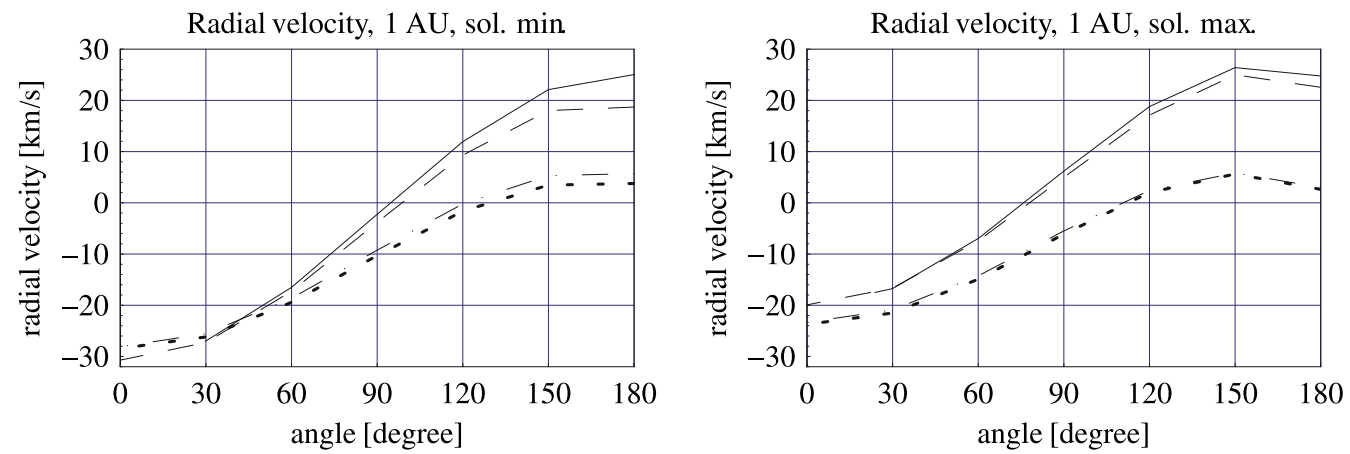

Fig. 7. Radial velocities of the primary and secondary populations of neutral $\mathrm{H}$ atoms at $1 \mathrm{AU}$ as a function of the offset angle from upwind for the solar minimum (left panel) and maximum conditions (right panel), calculated with the use of the Doppler and classical hot models. The upper pairs of lines in both panels correspond to the primary population and the lower pairs to the secondary population. The classical hot model results are drawn, correspondingly, with solid and dash-dot lines, and the Doppler model results with broken and dotted lines.
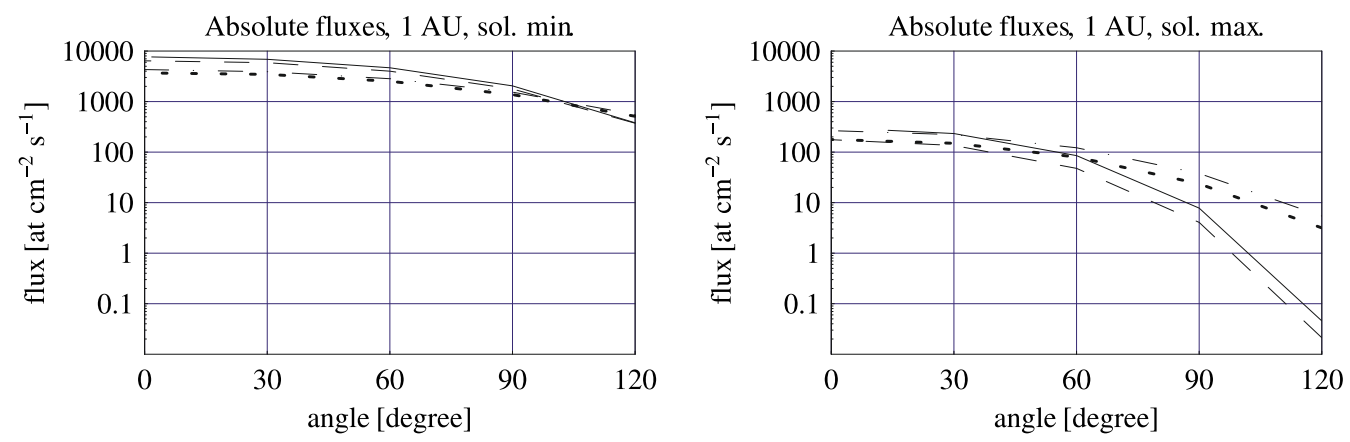

Fig. 8. Absolute fluxes of neutral interstellar $\mathrm{H}$ atoms at $1 \mathrm{AU}$ from the primary and secondary populations for the solar minimum (left panel) and solar maximum conditions (right panel), shown as a function of the offset angle from upwind, calculated with the use of the Doppler and classical hot models. The classical model results are drawn with solid line (primary populations) and dash-dotted line (secondary population); the Doppler model results with dashed (primary) and dotted lines (secondary).

density, which is directly proportional to the density at the termination shock. Because of this, the density at the termination shock derived from an in situ-measured flux at 1 AU neglecting the effect of $v_{\mathrm{r}}$ dependence of radiation pressure will be at least $15 \%$ off the mark (Fig. 9), and most probably more, up to a factor of 2 .

Another factor that can potentially affect interpretation of such measurements are differences in the local bulk velocity vectors of the two populations. Möbius et al. (2001) proposed to use the relative positions of the beams from the primary and secondary populations to check whether their flow directions at the termination shock are parallel or not. Such measurements will hopefully be realized soon by a forthcoming NASA SMEX mission IBEX (McComas et al. 2004, 2005, 2006). In Fig. 10 we show that the deflections of the two populations differ from the predictions of the wavelength-independent model. For both populations in the region of the Earth orbit where detection by IBEX is the most probable, the differences in deflection angle are only a few degrees and of a similar, but not identical magnitude. The magnitude of the deflection is a strong function of solar activity, with radiation pressure clearly playing the dominant role, but higher values are consistently returned by the Doppler model.

Even though the differences are just a few degrees, they cannot be regarded as negligible. Quémerais et al. (1999) and Lallement et al. (2005) suggested an upwind direction of neutral interstellar hydrogen that differs by about $5^{\circ}$ from the inflow direction of interstellar helium measured by Witte (2004). The difference is commonly attributed to a distortion of the 


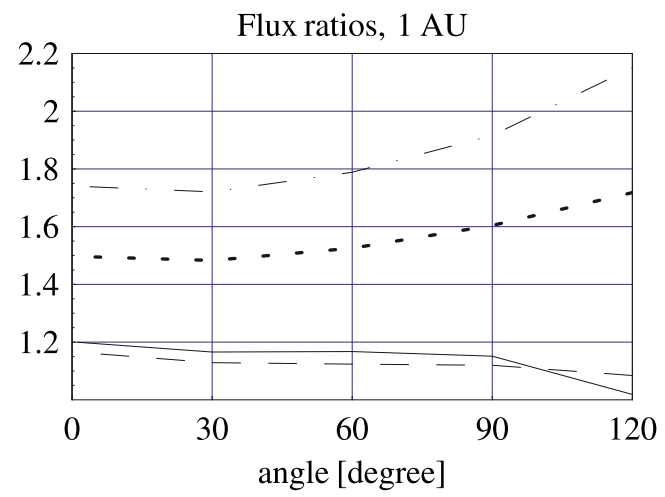

Fig. 9. Classical/Doppler ratios of the fluxes of the primary and secondary populations of neutral interstellar $\mathrm{H}$ atoms for the solar minimum and maximum conditions as a function of the offset angle from upwind. The lower pair of lines corresponds to solar minimum, the upper pair to solar maximum. Within the solar minimum (lower) pair, the solid line corresponds to the primary population, the broken line to the secondary population. Within the solar maximum (upper) pair, the dashdot line is for the primary, the dotted line for the secondary population.

heliospheric interface by the extraheliospheric magnetic field; hence mostly affected should be the secondary population and the difference in the upwind direction of the primary and secondary populations inferred from analysis of an IBEX-like in situ measurements at $1 \mathrm{AU}$ with the use of a $v_{\mathrm{r}}$-insensitive model might be erroneously attributed to a deformation of the interface. Our results show that the deflections of the secondary population obtained from the Doppler and classical models are very similar to each other, but the deflections of the primary population differ by a few degrees.

Finally, we assess the influence of the $v_{\mathrm{r}}$-sensitive radiation pressure on the expected PUI fluxes at 5 AU crosswind, i.e., the location where the $\mathrm{H}^{+}$PUI flux observed by Ulysses is the strongest. Bzowski et al. (2008) point out that the local production rate of pickup ions, which is a product of the local density of neutral hydrogen and of the local ionization rate, is a weak function of radiation pressure, hence, necessarily, of its details including the $v_{\mathrm{r}}$ dependence. They provide an estimate of the importance of the $v_{\mathrm{r}}$-dependence of radiation pressure for the local production rate of PUI, which suggests it is on the level of a few percent. Here we report its influence on the total flux of PUI, which was computed following the simple approach by Vasyliunas \& Siscoe (1976) and Gloeckler et al. (1993). Results of these simulations suggest that the classical hot model yields an excess flux with respect to the Doppler model, which at $\sim 5 \mathrm{AU}$ crosswind is equal to only $\sim 7.5 \%$ at solar minimum and to $8.5 \%$ at solar maximum. Thus we conclude that neglecting the $v_{\mathrm{r}}$ dependence of radiation pressure results in an overestimate of the $\mathrm{H}^{+}$PUI flux at $5 \mathrm{AU}$ from the Sun by about $10 \%$, irrespective of the solar activity phase.

\subsection{Comparison with the hot model: a scan of the parameter space}

The density excess $q$ is a weakly increasing and almost linear function of the ionization rate (Fig. 11); i.e., the higher the ionization rate taken, the lower the quality of the approximation given by the classical hot model obtained. This can be explained by preferential elimination of slower atoms from the local ensemble. As discussed by Lallement et al. (1985a) and Bzowski et al. (1997), ionization results in a net acceleration of the gas by a few $\mathrm{km} \mathrm{s}^{-1}$ because the atoms that have higher specific velocities in the ensemble survive against ionization better. Such atoms also have higher radial velocities, so they experience a higher radiation pressure due to the non-flat shape of the solar Lyman- $\alpha$ line profile. This force repels them from the Sun stronger than when the solar line profile is flat. As a result, we have a reduction in the density; consequently, the classical model predicts an excess of the density, whose magnitude increases with the increase in the ionization rate.

The sensitivity of the excess to the ionization rate increases with the offset angle from upwind and with the magnitude of the solar flux $I_{\text {tot }}$. At the upwind axis, the slope $\partial q / \partial \beta$ for an $I_{\text {tot }}$ value corresponding to the solar minimum conditions is equal to half of the value for crosswind and to $\sim 0.1$ of the value for downwind. For an $I_{\text {tot }}$ value related to the solar maximum conditions, the slope is steeper and the highest increase occurs at the upwind axis.

In the downwind region, increasing the ionization rate improves the quality of the approximation provided by the classical hot model with respect to the Doppler model. The parameter $q$ is still an increasing function of the ionization rate, but since it is lower than 1 in the downwind region, its increase means an improvement.

The magnitude of the excess $q$ is a weak function of the temperature and velocity of the gas at the termination shock, as shown in Fig. 12. At 1 AU crosswind, for $I_{\text {tot }}$ related to the solar minimum conditions, it varies from 1.15 to 1.30 , depending on the bulk velocity and temperature of the gas at the termination shock; for $I_{\text {tot }}$ related to the solar maximum conditions the amplitude is similar, but the value of $q$ higher.

Generally, the quality of the hot-model approximation of the density is consistently lower for higher values of $I_{\text {tot }}$, which is modulated by the solar activity level. The sensitivity of radiation pressure to the radial velocity thus modifies the relations between the densities during various phases of solar activity (the amplitude of modulation), as discussed, e.g., by Ruciński \& Bzowski (1995b); Bzowski \& Ruciński (1995a) and Bzowski et al. (2002).

On the other hand, it seems that the effect on the model values of the heliospheric Lyman- $\alpha$ glow should not be greater than $10 \%$. An exact calculation requires developing a model, where the local bulk velocity and temperature of the gas and their radial gradients would be taken into account, together with the wavelength dependence of the solar flux and its wavelengthdifferential attenuation, which increases with the increase in the solar distance. We believe this effect to be relatively weak because during solar minimum, when the contribution to the net backscatter intensity from the gas near the Sun is greatest, the $q$ factor is lowest. During solar maximum, when $q$ is greatest and hence the quality of the $v_{\mathrm{r}}$-independent model is lowest, the local density of the gas is very much reduced, so increased is the contribution to the net signal of more distant regions of the heliosphere, where the $q$ values are equal to 1 . In consequence, the $v_{\mathrm{r}}$ dependence of the radiation pressure should appreciably affect in situ measurements within $\sim 3$ to 5 AU from the Sun, but the photometric observations of the heliospheric glow only to a small degree.

\section{Conclusions}

We compared the density, bulk velocity, and flux of neutral interstellar hydrogen in the inner heliosphere, calculated using either the classical hot model or a newly-developed model with the 

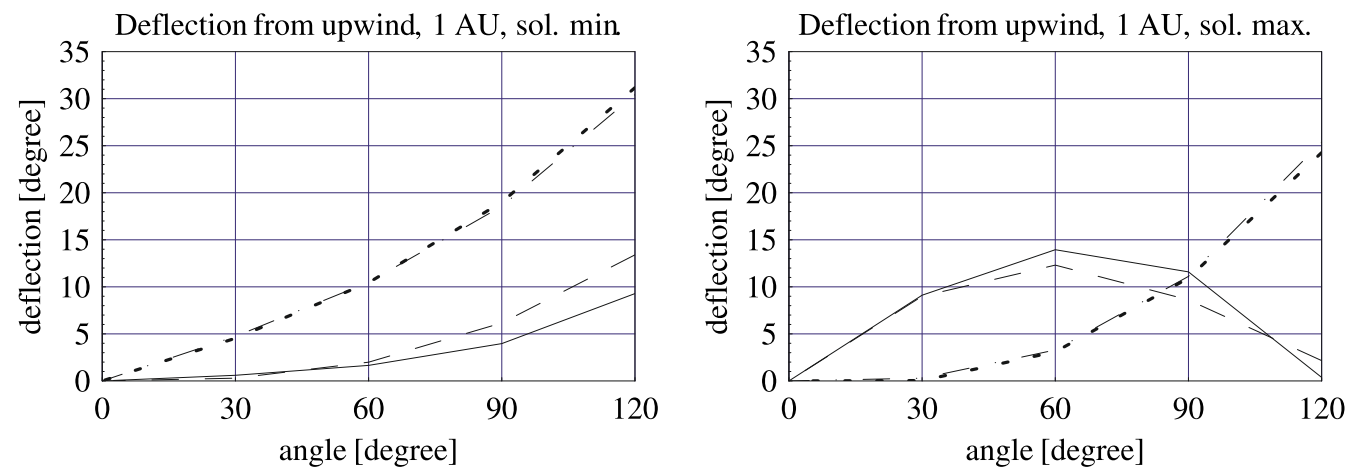

Fig. 10. Deflection of the local upstream direction of the primary and secondary populations of interstellar hydrogen at 1 AU as a function of the offset angle from the gas inflow direction (upwind) for solar minimum and maximum conditions. At solar minimum (left panel), the primary population is less deflected (solid line: classical model, broken line: Doppler model); the secondary is drawn with the dotted (Doppler) and dash-dotted line (classical). Same scheme for the solar maximum plot (right panel).
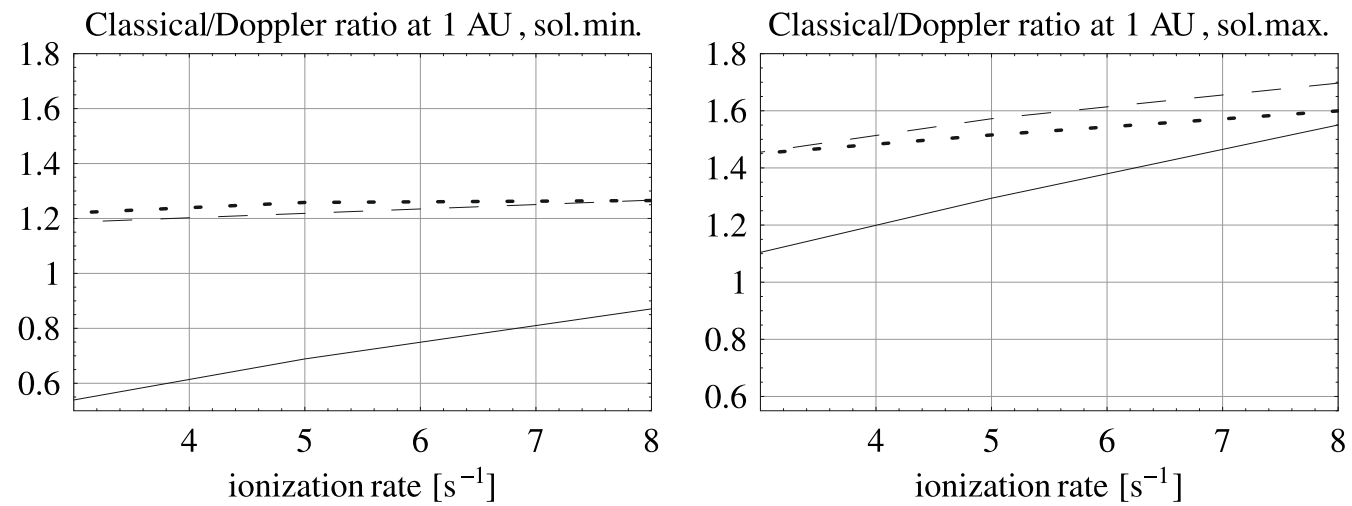

Fig. 11. Classical hot/Doppler model density ratios for the upwind (dots), crosswind (dashed), and downwind axes (solid) at 1 AU as a function of the ionization rate for the solar minimum (left panel) and maximum conditions (right panel).

Classical/Doppler ratio at crosswind, sol. min

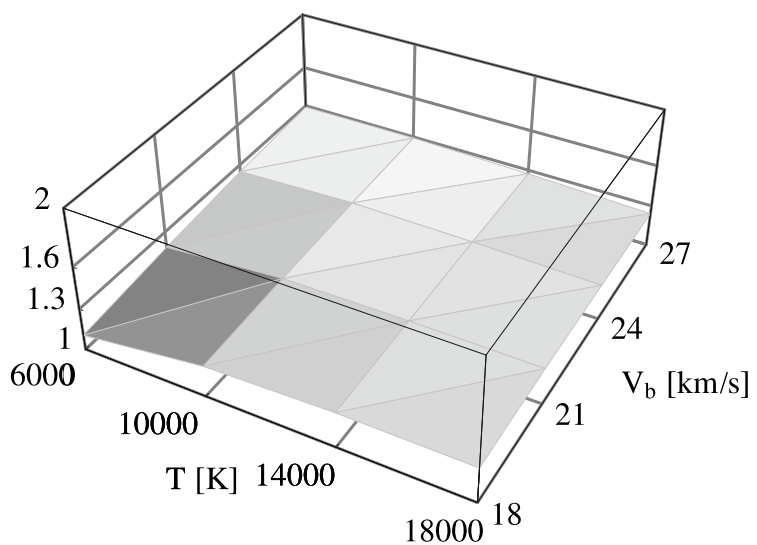

Classical/Doppler ratio at crosswind, sol. max.

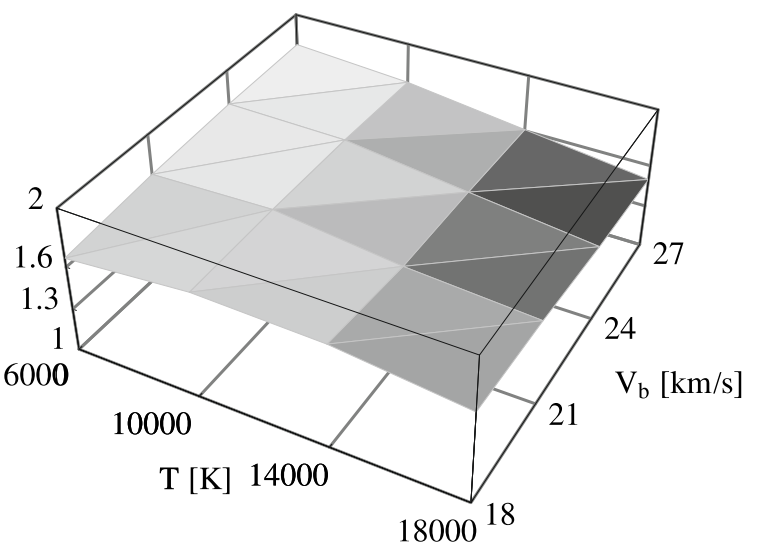

Fig. 12. Density excess $q$ of the classical hot model with respect to the Doppler model of neutral interstellar hydrogen as a function of the temperature and bulk velocity in the source region for the solar minimum (left panel) and solar maximum conditions (right panel).

radiation pressure being a function of specific radial velocities of individual atoms. The conclusions are the following.

1. Differences between the Doppler and classical hot models are restricted to $\sim 3$ to $\sim 5 \mathrm{AU}$ from the Sun, depending on the angle off the upwind direction. The classical hot model overestimates the density of neutral hydrogen gas everywhere in the inner heliosphere apart from a $\sim 60^{\circ}$ cone around the downwind direction, where a density deficit is predicted. Generally, the density excess/deficit is a strong function of the offset angle from the upwind direction and of the heliocentric distance (Figs. 3-5).

2. The magnitude of density excess $q$ varies with the solar activity level and is higher at solar maximum (Figs. 3 and 4), i.e. $v_{\mathrm{r}}$-independent models stronger overestimate the local density during solar maximum.

3. The density excess $q$ is a weakly increasing, almost linear function of the ionization rate in the inner heliosphere and a weak, almost bilinear function of the bulk velocity and temperature of the gas at the termination shock (Figs. 11 and 12). 
4. The classical model shows a higher upwind-downwind amplitude of radial velocities at $1 \mathrm{AU}$ than the Doppler model; the differences are about $10 \%$. The excess of the absolute flux of neutral atoms, returned by the classical model, is close to $15 \%$ at $1 \mathrm{AU}$ during solar minimum, i.e. at the time of observations by the forthcoming IBEX mission. The excess is much higher during solar maximum, but the absolute values of the fluxes are lower by a few orders of magnitude than during solar minimum (Figs. 7-9, 6).

5. The classical model returns a different deflection of the local upstream directions of the primary population of neutral interstellar $\mathrm{H}$ than the Doppler model. The deflections of the secondary population returned by the classical and Doppler models are practically identical, meaning that any interpretation of in situ measurements of these fluxes must be done very carefully, with an appropriate account of the solar line profile shape to avoid confusing deflections caused by the Doppler effect in the radiation pressure with, e.g., results of deformating the heliospheric interface by external magnetic field (Fig. 10).

6. Discrepancies between the PUI flux at Ulysses in aphelion, returned by the Doppler and classical models, are about $10 \%$ and are almost insensitive to the phase of the solar cycle.

Acknowledgements. M.B. gratefully acknowledges the hospitality of the ISSI Institute in Bern, where a part of this work was carried out within the framework of a Working Group on Heliospheric Breathing. This work was supported by Polish grants 1P03D00927 and N522 002 31/0902 and by SRC PAS PhD fellowship to S.T.

\section{References}

Amblard, P. O., Moussaoui, S., Dudok de Wit, T., et al. 2008, A\&A, 487, L13

Axford, W. I. 1972, in The Solar Wind, ed. J. M. W. C. P. Sonnet, P. \& J. Coleman, NASA Spec. Publ., 308, 609

Baranov, V. B. 2006a, in The physics of the heliospheric boundaries, ed. V. Izmodenov, \& R. Kallenbach, ISSI Sci. Rep, SR-005, 27

Baranov, V. B. 2006b, in The physics of the heliospheric boundaries, ed. V. Izmodenov, \& R. Kallenbach, ISSI Sci. Rep, SR-005, 1

Baranov, V. B., \& Malama, Y. G. 1993, J. Geophys. Res., 98, 15157

Baranov, V. B., Lebedev, M. G., \& Malama, Y. G. 1991, ApJ, 375, 347

Bertaux, J. L., \& Blamont, J. E. 1971, A\&A, 11, 200

Blum, P., \& Fahr, H. J. 1970, A\&A, 4, 280

Bzowski, M. 2001, in The Outer Heliosphere: The Next Frontiers, ed. K. Scherer, H. Fichtner, H. J. Fahr, \& E. Marsch (Pergamon: Elsevier), COSPAR Coll. Ser., 11, 69

Bzowski, M. 2008, A\&A, 488, 1057

Bzowski, M., \& Ruciński, D. 1995a, Space Sci. Rev., 72, 467

Bzowski, M., \& Ruciński, D. 1995b, Adv. Space Res., 16, 131

Bzowski, M., Fahr, H. J., Ruciński, D., \& Scherer, H. 1997, A\&A, 326, 396

Bzowski, M., Summanen, T., Ruciński, D., \& Kyrölä, E. 2001, in The outer heliosphere: the next frontiers, ed. K. Scherer, H. Fichtner, H. J. Fahr, \& E. Marsch (Pergamon: Elsevier), COSPAR Coll. Ser., 11, 129

Bzowski, M., Summanen, T., Ruciński, D., \& Kyrölä, E. 2002, J. Geophys. Res., 107, 10.1029/2001JA00141
Bzowski, M., Möbius, E., Tarnopolski, S., Izmodenov, V., \& Gloeckler, G. 2008, A\&A, 491, 7

Chabrillat, S., \& Kockarts, G. 1997, Geophys. Res. Lett., 24, 2659

Costa, J., Lallement, R., Quémerais, E., et al. 1999, A\&A, 349, 660

Emerich, C., Lemaire, P., Vial, J.-C., et al. 2005, Icarus, 178, 429

Fahr, H. J. 1968, Ap\&SS, 2, 474

Fahr, H. J. 1978, A\&A, 66, 103

Fahr, H. J. 1979, A\&A, 77, 101

Fahr, H. J., Ripken, H. W., \& Lay, G. 1981, A\&A, 102, 359

Floyd, L. E., Prinz, D. K., Crane, P. C., \& Herring, L. C. 2002, Adv. Space Res., 29, 1957

Gloeckler, G., Geiss, J., Balsiger, H., et al. 1993, Science, 261, 70

Izmodenov, V., Malama, Y. G., Gloeckler, G., \& Geiss, J. 2003, ApJ, 594, L59

Izmodenov, V. V. 2000, Ap\&SS, 274, 55

Izmodenov, V. V. 2006, in The physics of the heliospheric boundaries, ed. V. Izmodenov, \& R. Kallenbach, ISSI Sci. Rep, SR-005, 45

Izmodenov, V. V., \& Baranov, V. B. 2006, in The physics of the heliospheric boundaries, ed. V. Izmodenov, \& R. Kallenbach, ISSI Sci. Rep, SR-005, 67

Izmodenov, V. V., Gruntman, M. A., \& Malama, Y. G. 2001, J. Geophys. Res., 106,10681

King, J. H., \& Papitashvili, N. E. 2005, J. Geophys. Res., 110, 2104

Kyrölä, E., Summanen, T., \& Råback, P. 1994, A\&A, 288, 299

Lallement, R., Bertaux, J. L., \& Dalaudier, F. 1985a, A\&A, 150, 21

Lallement, R., Bertaux, J. L., \& Kurt, V. G. 1985b, J. Geophys. Res., 90, 1413

Lallement, R., Raymond, J. C., Bertaux, J.-L., et al. 2004, A\&A, 426, 867

Lallement, R., Quémerais, E., Bertaux, J. L., et al. 2005, Science, 307, 1447

Lemaire, P., Emerich, C., Vial, J.-C., et al. 2005, Adv. Space Res., 35, 384

Lemaire, P. L., Emerich, C., Vial, J. C., et al. 2002, in From Solar Min to Max: Half a Solar Cycle with SOHO, ESA SP-508, 219

Malama, Y., Izmodenov, V. V., \& Chalov, S. V. 2006, A\&A, 445, 693

McComas, D., Allegrini, F., Bartolone, L., et al. 2004, in Physics of the Outer Heliosphere, AIP Conf. Ser., 719, 162

McComas, D., Allegrini, F., Bartolone, L., et al. 2005, in Connecting Sun and Heliosphere, Solar Wind 11/SOHO 16, ESA SP, 592, 689

McComas, D. J., Allegrini, F., Bartolone, L., et al. 2006, in American Institute of Physics Conference Series, Physics of the Inner Heliosheath, ed. J. Heerikhuisen, V. Florinski, G. P. Zank, \& N. V. Pogorelov, 858, 241

McMullin, D. R., Bzowski, M., Möbius, E., et al. 2004, A\&A, 426, 885

Möbius, E., Litvinenko, Y., Saul, L., Bzowski, M., \& Ruciński, D. 2001, in The Outer Heliosphere: The Next Frontiers, ed. K. Scherer, H. Fichtner, H. J. Fahr, \& E. Marsch, COSPAR Coll. Ser. (Pergamon: Elsevier), 11, 69

Osterbart, R., \& Fahr, H. J. 1992, A\&A, 264, 260

Quémerais, E., Lallement, R., \& Bertaux, J.-L. 1992, A\&A, 265, 806

Quémerais, E., Bertaux, J.-L., Lallement, R., et al. 1999, J. Geophys. Res., 104, 12585

Ruciński, D., \& Fahr, H. J. 1989, A\&A, 224, 290

Ruciński, D., \& Fahr, H. J. 1991, Annales Geophys., 9, 102

Ruciński, D., \& Bzowski, M. 1995a, A\&A, 296, 248

Ruciński, D., \& Bzowski, M. 1995b, Adv. Space Res., 16, 121

Ruciński, D., \& Bzowski, M. 1996, Space Sci. Rev., 78, 265

Scherer, H., Bzowski, M., Fahr, H. J., \& Ruciński, D. 1999, A\&A, 342, 601

Scherer, H., Fahr, H. J., Bzowski, M., \& Ruciński, D. 2000, Ap\&SS, 274, 133

Tarnopolski, S. \& Bzowski, M. 2008, A\&A, 483, L35

Tarnopolski, S. T. 2007, Ph.D. Thesis, Space Research Centre PAS

Thomas, G. E. 1978, Ann. Rev. Earth Planet. Sci., 6, 173

Thomas, G. E., \& Krassa, R. F. 1971, A\&A, 11, 218

Tobiska, W. K., Woods, T., Eparvier, F., et al. 2000, J. Atm. Terr. Phys., 62, 1233

Vasyliunas, V., \& Siscoe, G. 1976, J. Geophys. Res., 81, 1247

Vidal-Madjar, A. 1975, Sol. Phys., 40, 69

Wilhelm, K., Woods, T. N., Schühle, U., et al. 1999, A\&A, 352, 321

Witte, M. 2004, A\&A, 426, 835

Wu, F. M., \& Judge, D. L. 1979, ApJ, 231, 594 